\title{
Editorial
}

\section{Welcome to the New Era of Inflammation and Regeneration}

On behalf of the editorial board and editors, I would like to thank you for your interest and contribution to this journal. It is my great pleasure to announce the renewal of Inflammation and Regeneration as an international online journal open to scientists working in this field. As all of you have noticed, interest in the topics of inflammation and regeneration has been increasing. Inflammation is characteristic of the pathophysiologies of various diseases including autoimmune diseases, infectious diseases, cancer, neurological diseases and even psychiatric disorders. Thus, a basic understanding and the appropriate management of inflammation are definitely beneficial for the treatment of these diseases. The rapid progress of research on regeneration/regenerative medicine, including stem cell biology, is also clearly apparent. For example, a basic understanding of the pluripotency of embryonic stem cells (ES cells) on a molecular level has led to the development of technology for inducing pluripotent stem cells (iPS cells), contributing definitively to medical science in terms of cell therapy and disease models. Furthermore, iPS technology has also provided a paradigm shift for the development of new drugs. Here, I wish to emphasize that both inflammatory research and regenerative medicine have not developed independently from each other, but in a mutually interactive fashion. To say that research on inflammation and regeneration has reached its most exciting stage ever would not be an exaggeration. Thus, I have no doubt that the renewal of Inflammation and Regeneration will make an enormous contribution to progress in this field of research. I eagerly welcome new and exciting papers to this journal.

Thank you very much for your cooperation.

\section{Hideyuki Okano}

Editor-in-Chief of Inflammation and Regeneration 